\title{
A Comparison of Solar Cycle Variations in the Equatorial Rotation Rates of the Sun's Subsurface, Surface, Corona, and Sunspot Groups
}

\author{
J. Javaraiah \\ (C) Springer $\bullet \bullet \bullet$
}

\begin{abstract}
Using the Solar Optical Observing Network (SOON) sunspot-group data for the period 1985-2010, the variations in the annual mean equatorialrotation rates of the sunspot groups are determined and compared with the known variations in the solar equatorial-rotation rates determined from the following data: i) the plasma rotation rates at $0.94 \mathrm{R}_{\odot}, 0.95 \mathrm{R}_{\odot}, \ldots, 1.0 \mathrm{R}_{\odot}$ measured by Global Oscillation Network Group (GONG) during the period 1995-2010, ii) the data on the soft X-ray corona determined from Yohkoh/SXT full disk images for the years $1992-2001$, iii) the data on small bright coronal structures (SBCS) which were traced in Solar and Heliospheric Observatory (SOHO)/EIT images during the period 1998-2006, and iv) the Mount Wilson Doppler-velocity measurements during the period $1986-2007$. A large portion (up to $\approx 30^{\circ}$ latitude) of the mean differential-rotation profile of the sunspot groups lies between those of the internal differential-rotation rates at $0.94 R_{\odot}$ and $0.98 R_{\odot}$. The variation in the yearly mean equatorial-rotation rate of the sunspot groups seems to be lagging that of the equatorial-rotation rate determined from the GONG measurements by one to two years. The amplitude of the latter is very small. The solar-cycle variation in the equatorial-rotation rate of the solar corona closely matches that determined from the sunspot-group data. The variation in the equatorial-rotation rate determined from the Mount Wilson Doppler-velocity data closely resembles the corresponding variation in the equatorial-rotation rate determined from the sunspot-group data that included the values of the abnormal angular motions $\left(>\left|3^{\circ}\right|\right.$ day $\left.^{-1}\right)$ of the sunspot groups. Implications of these results are pointed out.
\end{abstract}

\section{Introduction}

Studies on the solar-cycle variations in the solar differential rotation and meridional flow are important for understanding the physical system that generates solar activity and the solar cycle (Babcock, 1961; Ulrich and Boyden, 2005. Dikpati and Gilman, 2006, Karak, 2010). The solar differential rotation is well

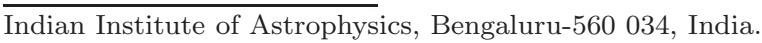
email: jj@iiap.res.in 
studied, in addition to the use of the Doppler-velocity measurements, using the data on different solar magnetic tracers (mainly the sunspots and the sunspot groups). The results derived from the sunspot and the sunspot-group data show variations in both the equatorial-rotation rate and the latitude gradient of the rotation on several time scales, including time-scales close to the 11-year solar cycle (Javaraiah and Gokhale, 1995 Javaraiah and Komm, 1999: Javaraiah, Bertello, and Ulrich, 2005a: Javaraiah, Bertello, and Ulrich, 2005b| Javaraiah and Ulrich, 2006 Brajša, Ruždjak, and Wöhl, 2006) Recently, Javaraiah (2011) detected variations on time-scales of a few days, including 9 and 30-40 day quasi-periodicities, in the coefficients of differential rotation determined from the Mount Wilson Doppler-velocity data during Solar Cycle 22 . The $\approx 11$-year period torsional oscillation detected by Howard and LaBontel (1980) from the Mount Wilson Doppler-velocity measurements is confirmed by the helioseismic studies (e.g., Howe et al., 2000: Antia, Basu, and Chitre, 2008)

Javaraiah and Komm (1999) studied the variations in the coefficients of the differential rotation determined from the sunspot group data (1879-1976) and the Mount Wilson Doppler-velocity data $(1962-1994)$. They found considerable differences between the periodicities ( $>$ two years) in the rotational coefficients derived from the Doppler-velocity and the sunspot-group data. Javaraiah et al. (2009) analyzed the data on the solar surface equatorial-rotation rate derived from the more accurate Mount Wilson Doppler-velocity data during the period $1986-2007$ and confirmed the short-term periodicities found by Javaraiah and Komm (1999) in the data before the year 1996, but found no statistically significant variation after 1996. That is, there is a very large difference between the temporal variations of the equatorial-rotation rate during Solar Cycle 22 (i.e. before 1995) and Cycle 23. Hence, it has been suspected that the frequent changes in the instrumentation of the Mount Wilson spectrograph might have made the data before 1995 erroneous and responsible for the variations in the equatorialrotation rate derived from this data. To verify this, here we compare the variations in the equatorial- rotation rates derived from different rotational data and by using different techniques.

\section{Data and Analysis}

\subsection{METHODOLOGY}

The solar differential rotation can be determined from the full-disk Dopplervelocity data using the traditional polynomial expansion

$$
\omega(\phi)=A+B \sin ^{2} \phi+C \sin ^{4} \phi,
$$

and from sunspot data by using the first two terms of the expansion, i.e.

$$
\omega(\phi)=A+B \sin ^{2} \phi,
$$

where $\omega(\phi)$ is the solar sidereal angular velocity at heliographic latitude $\phi$, the coefficient $A$ represents the equatorial-rotation rate, and $B$ and $C$ measure the 
latitudinal gradient in the rotation rate with $B$ mainly representing low latitudes and $C$ mainly higher latitudes.

The sidereal rotation rate $\left[\omega\right.$, in degrees $\left.d^{-1} y^{-1}\right]$ of a sunspot or sunspot group is computed as (Carrington, 1863 Balthasar, Vázquez, and Wöhl, 1986. Godoli and Mazzucconi, 1979):

$$
\omega=\frac{\Delta L}{\Delta t}+14.18
$$

or as (e.g. Howard, Gilman, and Gilman, 1984, Kambry and Nishikawa, 1990, Gupta, Sivaraman, and Howard, 1999, Brajša, Ruždjak, and Wöhl, 2006):

$$
\omega=\frac{\Delta D_{C M}}{\Delta t}+0.9856
$$

where $\Delta L, \Delta D_{\mathrm{C} M}$, and $\Delta t$ are the differences between the heliographic longitudes $[\mathrm{L}]$, the central meridian distances $\left[D_{\mathrm{C} M}\right]$, and the observation times $[t]$ of two consecutive days observations of the sunspot or sunspot group, respectively, and the values $14.18^{\circ}$ day $^{-1}$ and 0.9856 are the Carrington rigid-body rotation rate and the correction factor corresponding to the Earth's rotation, respectively. The quantity $\frac{\Delta D_{\mathrm{C} M}}{\Delta t}$ represents the synodic rotation rate. Within the uncertainties, both these methods yield the same result (the latter gives a relatively small value for the rotation rate; see Kambry and Nishikawa, 1990). Javaraiah and co-authors have used Equation (3) in all of their earlier articles (e.g. Javaraiah and Gokhale, 1995, 1997a, 1997b, Javaraiah and Komm, 1999, Javaraiah, 2003a, 2005, Javaraiah, Bertello, and Ulrich, 2005a, 2005b; Javaraiah and Ulrich, 2006). (Details on the method of conversion of the synodic to sidereal rotation rate can be found in some of the above cited articles, e.g. Brajša, Ruždjak, and Wöhl] 2006 and references therein.)

The solar differential rotation can also be studied by binning the data into latitude intervals of reasonably small size, i.e. without using the above equations (see the references above). This subject is reviewed by a number of authors (e.g. Javaraiah and Gokhale, 2002 and references therein).

\subsection{SUNSPOT GROUP DATA}

Here the Greenwich sunspot-group data during period May/1874-December/1976 and the SOON sunspot-group data during 1977-2010 are used. These data are taken from the website http://solarcience.msfc.nasa.gov/green wich.shtml, and consist of $t, \phi, L, D_{\mathrm{C} M}$, etc., for each sunspot group on each day of its observation. By using Equation (3), the $\omega$ values are determined from the data corresponding to each pair of the consecutive days' observations of the sunspot groups during the period 1986-2009. Each year's data are fitted to Equation (2). The latitudinal dependencies in the mean rotation rate of the sunspot groups over the whole period 1977-2011 (whole SOON data set) is determined by averaging the daily values of the rotational velocities in twodegree (and $5^{\circ}$ ) latitude intervals and also by fitting the whole period daily data to Equation (2). The data in both the Northern and the Southern hemispheres 
have been combined. These calculations are also done by using the Greenwich data during the period May 1874-December 1976.

Since the rotation rates of tracers depend on the lifetimes/sizes/age of the tracers, the latitudinal dependence in the initial rotation rates (the first two days' heliographic positions of the sunspot groups are used) of the sunspot groups is also studied by classifying the sunspot groups on the basis of their lifetimes: one-three days, four-five days, six-eight days, and $>$ eight days. For this the combined Greenwich and SOON data are used.

The sunspot-group data that correspond to $\left|D_{\mathrm{C} M}\right|>75^{\circ}$ in any day of the sunspot group lifetime are not used. In the case of the study of the initial rotation rates of the sunspot groups, in order to avoid ambiguity in the identifications of the first two days in the lifetimes of the sunspot groups, the data during the entire lifetime of a sunspot group are eliminated even in any one day $\left|D_{\mathrm{C} M}\right|>75^{\circ}$. Since the cutoff of $D_{C M}$ is applied to both the Eastern and the Western sides, the sunspot groups that emerged at the visible side of the Sun and after certain number of days went to the other side were not taken into account in the data sample of any of the aforementioned classes (Note: each disk passage of a recurrent sunspot group is treated as an independent sunspot group). As in our previous articles, the data correspond to $>2^{\circ}$ day $^{-1}$ latitudinal motions and $>3^{\circ}$ day $^{-1}$ longitudinal motions (i.e. abnormal $\omega$ values) have been excluded. Ward (1965, 1966) found that this precaution substantially reduces the uncertainties in the results (see Javaraiah and Gokhale, 1995). However, the abnormal values of $\omega$ may have some physical significance. For example, the abnormal rotation rates of sunspot groups may play some role in the productions of solar flares (Hiremath and Suryanarayana, 2003; Suryanarayana, 2010). Hence, most of the above calculations are done for both the cases, i.e., both the sets of the sunspot-group data with and without the abnormal $\omega$ values included are used.

\subsection{MOUNT WILSON DOPPLER VELOCITY DATA}

The daily values of the equatorial-rotation rate $A$ derived from the Mount Wilson Doppler measurements during the period 3 December 1985 to 5 March 2007 are available (Javaraiah et al., 2009, This period covers Solar Cycle 22 and most of Cycle 23. The data have been corrected for scattered-light (for details see Ulrich, 2001). Javaraiah et al. (2009) used this data after removing the very large spikes (i.e. $>2 \sigma$, where $\sigma$ is the standard deviation of the original time series) and studied short-term variations in the corrected data. The time series has data gaps that vary in size, with a maximum gap of 49 days during Carringtonrotation numbers $1560-1608$. Therefore, the daily data were binned to one-year consecutive intervals and the annual average values of the equatorial-rotation rate (average of the daily values of $A$ over each year) were determined. In Figure 1 of Javaraiah et al. (2009) both the original and the corrected time series of the equatorial-rotation rate were shown. In the present analysis the corrected time series is used.

\subsection{DATA ON SUBSURFACE AND CORONAL ROTATION RATES}

The Sun's internal-rotation rates determined from the GONG data for 147 intervals of three GONG-months (a GONG-month is 36 days) which began on 7 May 


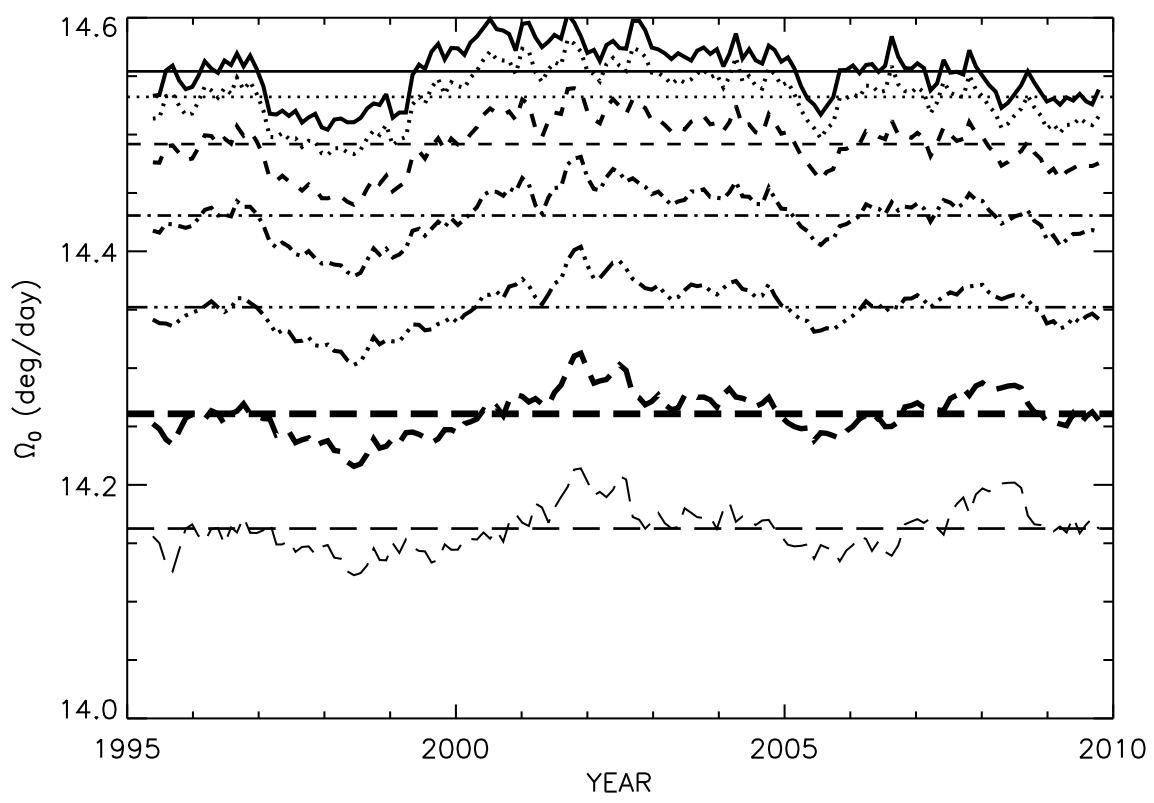

Figure 1. The solid, dotted, short-dashed, one dotted-dashed, three dotted-dashed, thick long-dashed, and thin long-dashed curves, represent the variations in the equatorial-rotation rate $\left(\Omega_{0}\right)$ at $0.94 \mathrm{R}_{\odot}, 0.95 \mathrm{R}_{\odot}, \ldots, 1.0 \mathrm{R}_{\odot}$ (in the order of decreasing mean values of $\Omega_{0}$ ), respectively, determined from the GONG data for 147 intervals of three GONG-months (for details about this data see Antia and Basu, 2010 and references therein).

1995 and ended on 31 October 2009 , for $0.005 \mathrm{R}_{\odot}, 0.01 \mathrm{R}_{\odot}, 0.015 \mathrm{R}_{\odot}, \ldots, 0.995 \mathrm{R}_{\odot}$, $1.0 \mathrm{R}_{\odot}$, and for latitudes $0,2,4, \ldots, 88$ degrees are available Antia and Basu, 2010). Figure 1 shows variations in the equatorial-rotation rate $\left[\Omega_{0}\right.$, angular velocity at latitude $\phi=0]$ at $0.94 \mathrm{R}_{\odot}, 0.95 \mathrm{R}_{\odot}, \ldots, 1.0 \mathrm{R}_{\odot}$. As can be seen in this figure the temporal patterns of the equatorial-rotation rates at the different depths are largely similar. However, there is a suggestion that some features are smoothed when going from interior towards the surface and vice versa. Specifically, the maxima in 2002 and 2008 are smoothed in deeper layers while the minimum in 2005 is smoothed from interior towards the surface.

The annual mean values of the equatorial-rotation rate of the soft X-ray corona determined from the Yohkoh/SXT solar full-disk images for the period 1992-2001 are taken from the Table 2 of Chandra, Vats, and Iyer (2010) and those determined from the rotation rates of small bright coronal structures (SBCS) that were traced in SOHO/EIT images during the period 1998-2006 are taken from Figure 1 of Jurdana-Šepić et al. (2011). 


\section{Results}

\subsection{DEPTH DEPENDENCE IN THE DIFFERENTIAL ROTATION OF SUNSPOT GROUPS}

Figure 2 shows the latitudinal dependence in the mean rotation rate of sunspot groups determined from the SOON sunspot-group data during the period 1977 2011 , separately from the data sets with and without the abnormal $\omega$ values included. In the same figure the latitudinal dependencies in the mean (over the whole period 1995-2009) rotation rates of the plasma at different depths of the solar convection zone deduced from the GONG data are also shown. As can be seen in this figure, a large portion (up to $\approx 30^{\circ}$ latitude) of the rotational profile that is obtained from the sunspot-group data that do not include the abnormal $\omega$ values lies between the corresponding profiles at $0.94 R_{\odot}-0.98 R_{\odot}$ determined from the GONG data. (Note: The value of the correlation coefficient shown in these figures is almost the same for any profile of the internal rotation shown in these figures.) As can be seen in Figure 3, the similar conclusion can also be drawn from the analysis of the large set of Greenwich data during May 1874-December 1976 (however, the helioseismic measurements are not exist before 1995). The latitude dependence in the rotation determined from the sunspot group data that included the abnormal $\omega$ values is obviously highly unrealistic. Since the equatorial-rotation rate determined from these data seems to be somewhat closer to the $\Omega_{0}$ at $1.0 \mathrm{R}_{\odot}$, the comparison between its variation and that of the surface is studied here.

There are more abnormal values in SOON data (5.8\%) than in Greenwich data $(2.9 \%)$. In the case of Greenwich data the observation time contains the date with the fraction of a day. In SOON data the fraction is rounded to 0.5 day. This might some extent increased the number of abnormal $\omega$ values in SOON data. Figure 4 shows the latitudinal dependence of the rotation determined by binning the sunspot-group data that included the abnormal $\omega$ values into $5^{\circ}$ latitude intervals. Obviously, the results shown in this figure the errors are relatively much smaller than those shown in the Figures 2(b) and (3(b). However, the latitudinal patterns of the data in $5^{\circ}$ latitude intervals (represented by the open circles) are largely same as the corresponding patterns of the data in $2^{\circ}$ latitude intervals (cf., Figures 2(b) and 3(b)). The Greenwich data that included the abnormal $\omega$ values show rigid-body rotation (this is more clear in Figure 4(b) than in Figure 3(b)).

On average the rotation rate determined from the magnetic tracers is larger than that derived from the Doppler-velocity measurements. This difference can be interpreted as the Doppler-velocity measurements representing the surface gas motion and the tracers motions representing the motions of the deeper layers (Foukal, 1972, Meunier, 2005), where the magnetic structures of the tracers are anchored. Besides the concept "anchoring of the magnetic structures of sunspot groups to the subsurface layers", the faster rotation rates of sunspots, compared to the photospheric plasma, have been explained in several ways: i) the emergent motion of the magnetic flux loop driven by buoyancy (e.g. Moreno-Insertis, 1986. Chou and Fisher, 1989, Shibata et al., 1990), ii) geometrical projection 

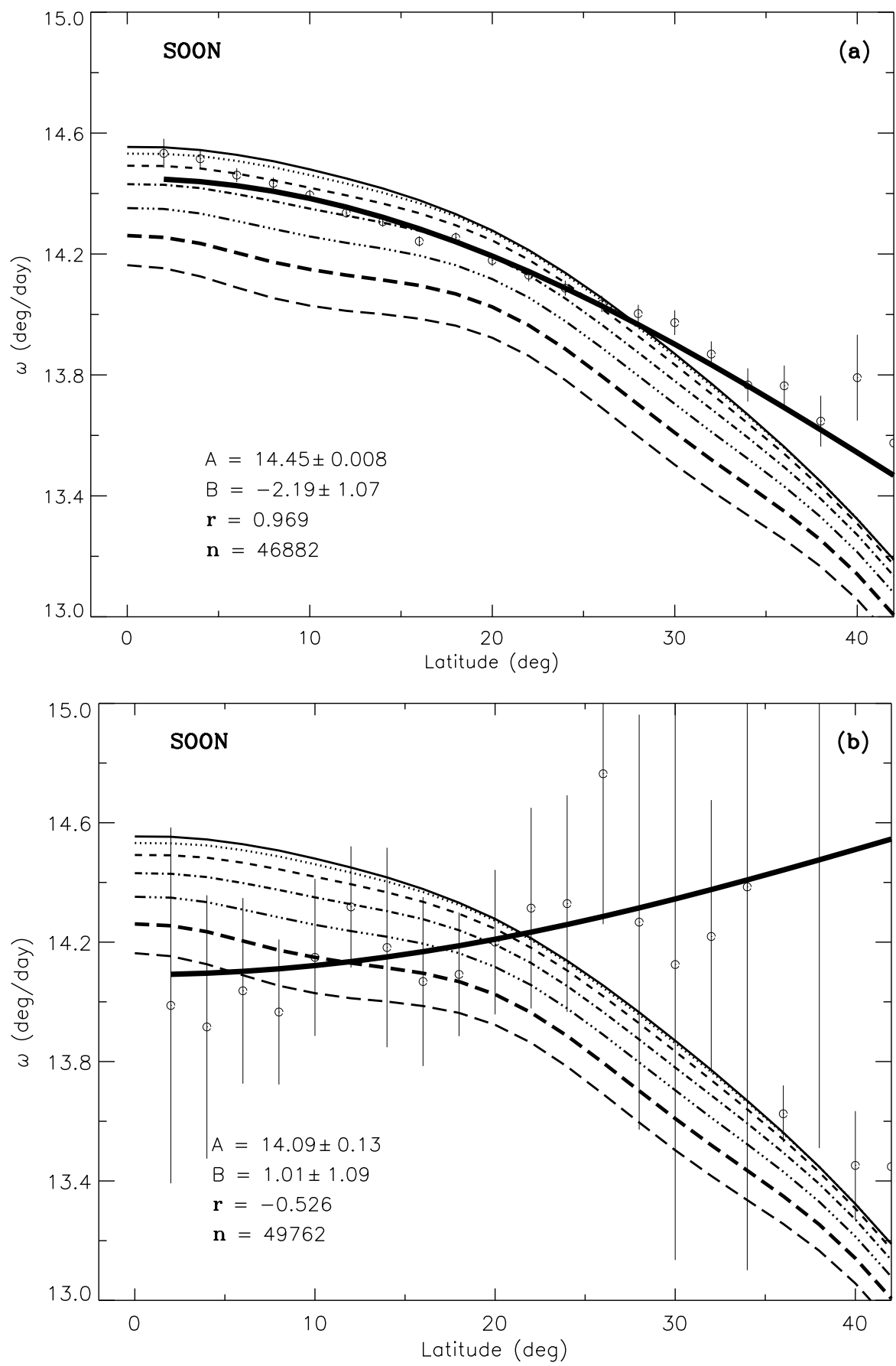

Figure 2. The latitudinal dependence of the mean rotation rates of sunspot groups determined by averaging the daily values of $\omega$ obtained from the SOON sunspot-group data during $1977-2011$ over $2^{\circ}$ latitude intervals, $1^{\circ}-3^{\circ}, 3^{\circ}-5^{\circ}, 5^{\circ}-7^{\circ}, \ldots, 40^{\circ}-42^{\circ}$ (plotted at $2^{\circ}$, $4^{\circ}, 6^{\circ}, \ldots, 42^{\circ}$ ). The error bars represent the standard errors. Panels (a) and (b) represent the data with and without the abnormal values of $\omega$, respectively. The thick solid curve represents the corresponding mean profile deduced from the values (also shown) of the coefficients $A$ and $B$ of Equation (2) obtained from the total number of daily data $[n]$. The different type curves represent the latitudinal dependencies in the mean (over the whole period 1995-2009) plasma rotation rates deduced from the GONG data at the same depths as in Figure 1. $r$ represents the coefficient of correlation between the sunspot group and the GONG data around $0.96 \mathrm{R}_{\odot}$ in $2^{\circ}, 4^{\circ}, 6^{\circ}, \ldots, 36^{\circ}$ latitudes. 

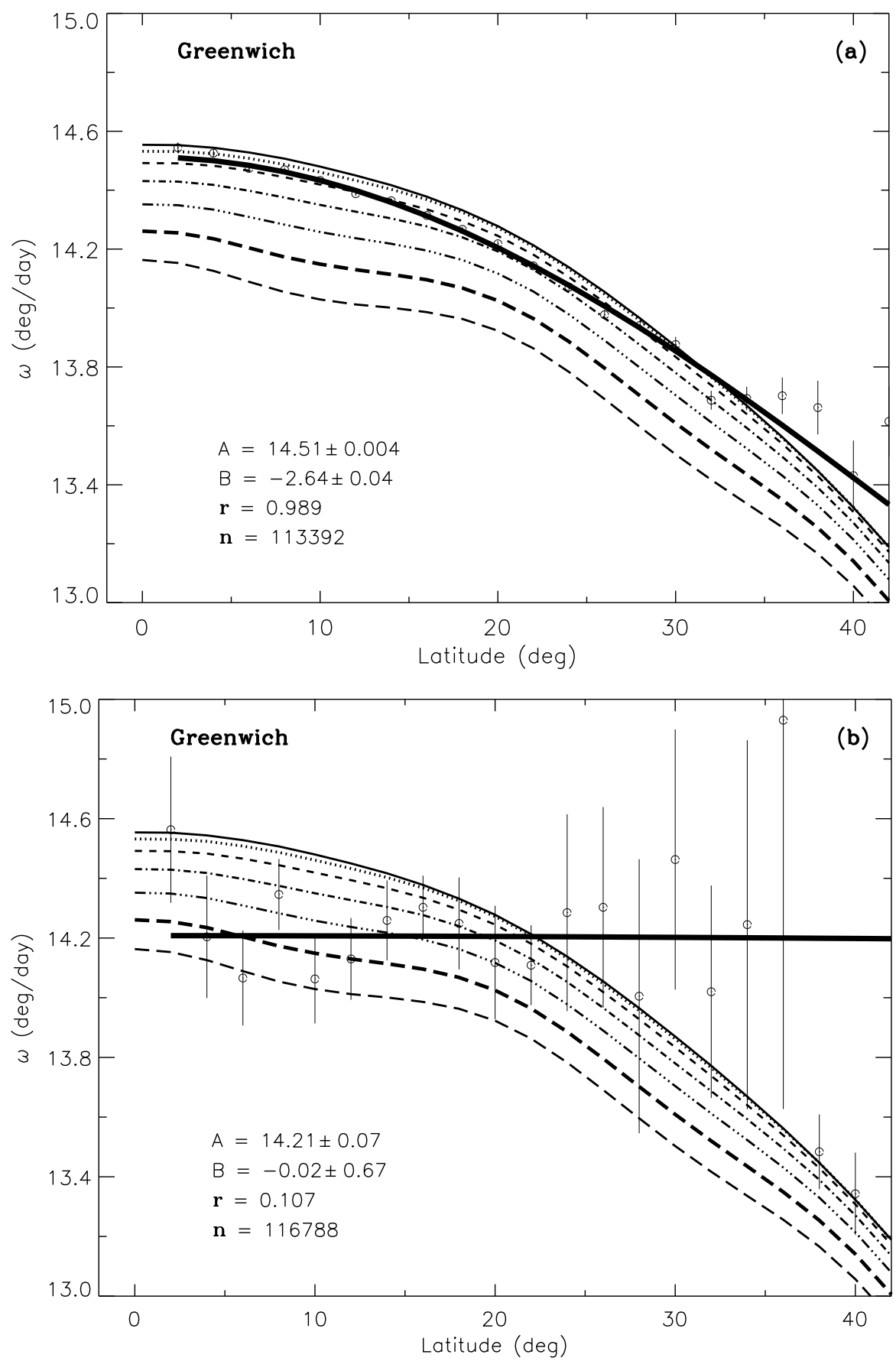

Figure 3. The latitudinal dependence of the mean rotation rates of sunspot groups determined by averaging the daily values of $\omega$ obtained from the Greenwich data during the period May $1874-$ December 1976 over $2^{\circ}$ latitude intervals, $1^{\circ}-3^{\circ}, 3^{\circ}-5^{\circ}, 5^{\circ}-7^{\circ}, \ldots, 40^{\circ}-42^{\circ}$ (plotted at $2^{\circ}, 4^{\circ}, 6^{\circ}, \ldots, 42^{\circ}$ ). The error bars represent the standard errors. Panels (a) and (b) represent the data with and without the abnormal values of $\omega$, respectively. The thick solid curve represents the corresponding mean profile deduced from the values (also shown) of the coefficients $A$ and $B$ of Equation (2) obtained from the total number of daily data $[n]$. The different type curves represent the latitudinal dependencies in the mean (over the whole period 1995-2009) plasma rotation rates deduced from the GONG data at the same depths as in Figure 1. $r$ represents the coefficient of correlation between the sunspot group and the GONG data around $0.96 \mathrm{R}_{\odot}$ in $2^{\circ}, 4^{\circ}, 6^{\circ}, \ldots, 36^{\circ}$ latitudes. 

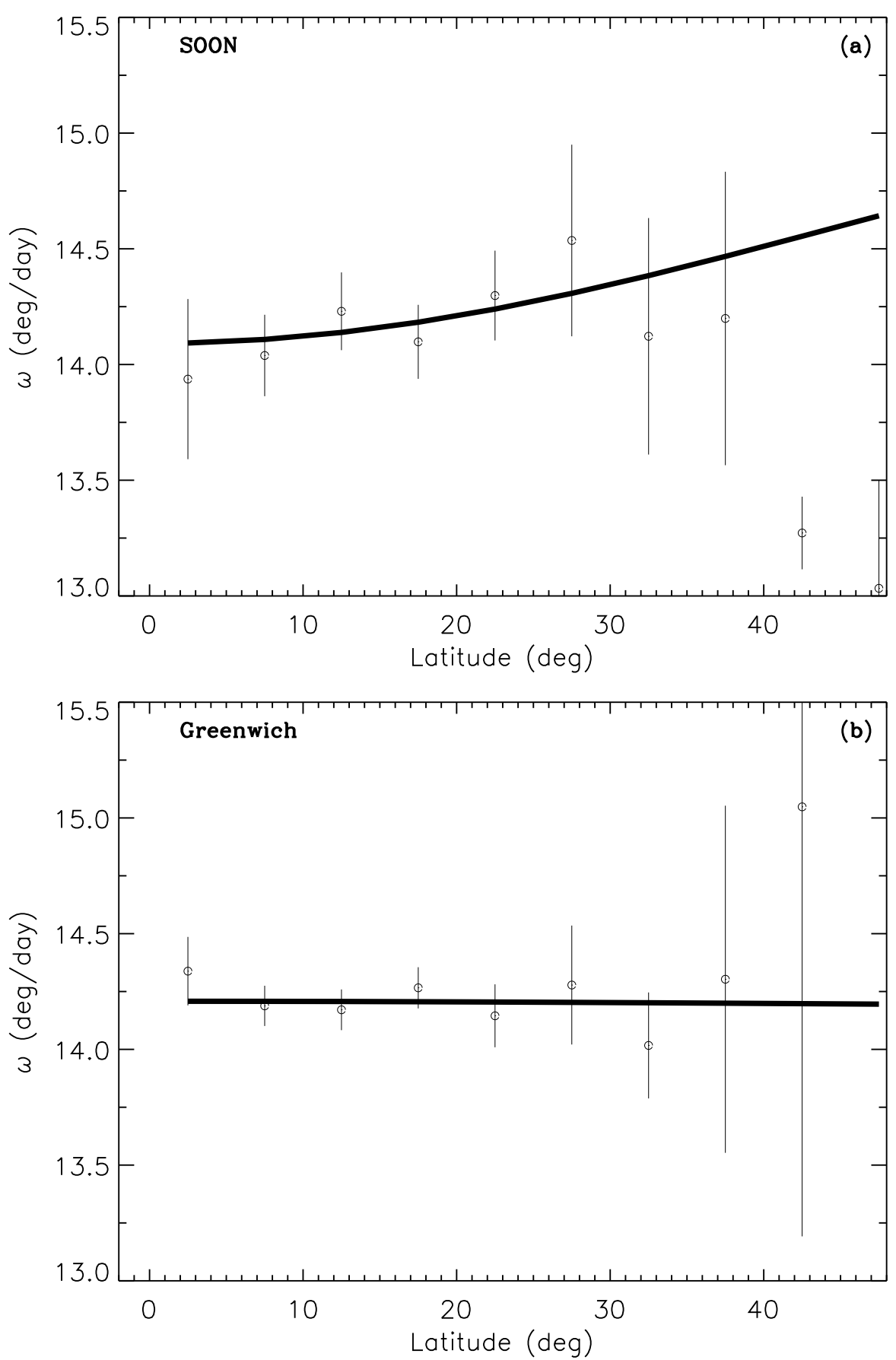

Figure 4. The latitudinal dependence of the mean rotation rates of sunspot groups determined by averaging the daily values of $\omega$, obtained from the SOON (1977-2011) and Greenwich (1874-1976) sunspot-group data that included the abnormal values of $\omega$, over $5^{\circ}$ latitude intervals, $0^{\circ}-5^{\circ}, 5^{\circ}-10^{\circ}, 10^{\circ}-15^{\circ}, \ldots, 45^{\circ}-50^{\circ}$ (plotted at $2.5^{\circ}, 7.5^{\circ}, 12.5^{\circ}, \ldots, 47.5^{\circ}$ ). The error bars represent the standard errors. The thick solid curve represents the corresponding mean profile deduced from the values of the coefficients $A$ and $B$ of Equation (2) obtained from the total number of daily data (i.e. the corresponding values that are given in Figures 2(b) and $3(\mathrm{~b}))$. 

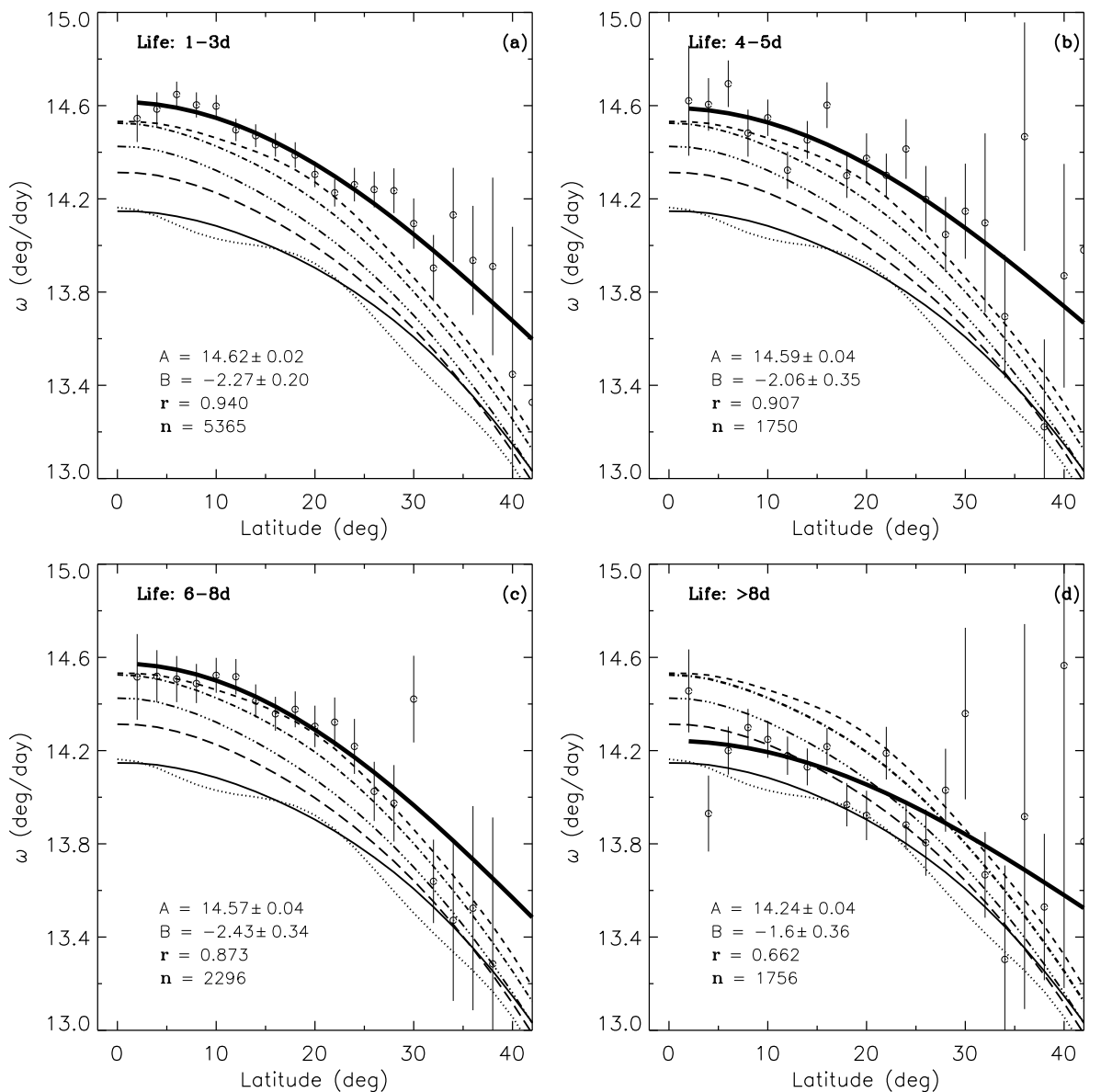

Figure 5. The latitudinal dependence of the "mean initial rotation rates" of sunspot groups whose life times are in the ranges: (a) one-three days, (b) four-five days, (c) six-eight days, and $(\mathrm{d})>$ eight days, determined from the combined Greenwich and SOON data during the period May 1874 -December 2011 by averaging the first day values of $\omega$ over $2^{\circ}$ latitude intervals, $1^{\circ}-3^{\circ}, 3^{\circ}-5^{\circ}, 5^{\circ}-7^{\circ}, \ldots, 40^{\circ}-42^{\circ}$ (plotted at $2^{\circ}, 4^{\circ}, 6^{\circ}, \ldots, 42^{\circ}$ ). The error bars represent the standard errors. The thick solid curve represents the corresponding mean profile deduced from the values (also shown) of the coefficients $A$ and $B$ of Equation (2) obtained from the total number of daily data $[n]$. Here the internal-rotational profiles are correspond to the different depths: thin-solid, long-dashed, three-dotted-dashed, one-dotted-dashed, dashed, and dotted curves represent the profiles at depths $0.75 \mathrm{R}_{\odot}, 0.80 \mathrm{R}_{\odot}, 0.85 \mathrm{R}_{\odot}, 0.90 \mathrm{R}_{\odot}, 0.95 \mathrm{R}_{\odot}$, and $1.0 \mathrm{R}_{\odot}$, respectively. (Note: the sunspot-group data corresponding to $\left|D_{\mathrm{C} M}\right| \leq 75^{\circ}$ are used.)

effects (van Driel-Gesztelyi and Petrovay, 1990), iii) the drag due to ambient flows (Meyer et al., 1979, Petrovay et al., 1990), and iv) the interaction between magnetic buoyancy, drag, and Coriolis forces acting on the rising flux tubes (D'Silva and Howard, 1994). However, in spite of the aforementioned effects, the similarity between the variation in the initial rotation rates of the sunspot groups with their lifetimes and the radial variation of the internal rotation rate determined from helioseismology 

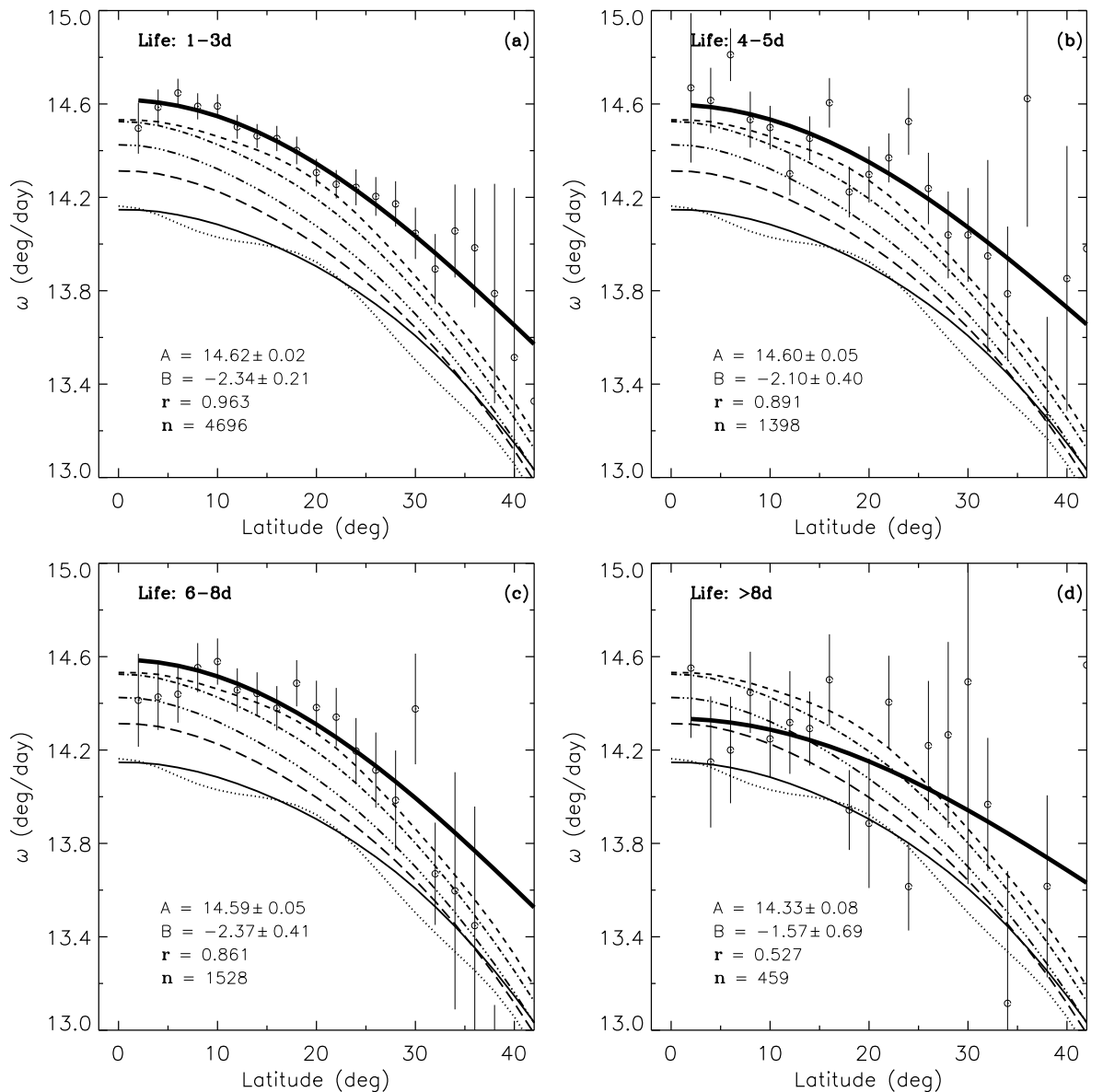

Figure 6. The same as Figure 5, but for the sunspot-group data corresponding to $\left|D_{C M}\right| \leq 70^{\circ}$.

suggests that the magnetic structures of sunspot groups with successively longer life times (2-12 days) are initially anchored in successively deeper layers of the Sun's convection zone (Javaraiah and Gokhale, 1997b). Hiremath (2002) confirmed this result by analyzing a large set of the Greenwich sunspot-group data and Sivaraman et al. (2003) confirmed it by analyzing the sunspot-group data measured at the Mount Wilson and Kodaikanal Observatories. Short-lived/small sunspot groups predominate in a given time interval (Javaraiah, 2012). Moreover, in the low latitudes near the base of the convection zone to near $0.8 \mathrm{R}_{\odot}$ the internal rotation rate steeply increases and then it gradually increases (or remains almost constant) up to near $0.95 \mathrm{R}_{\odot}$. The magnetic structures of the sunspot groups whose lifetimes exceed eight days seem to initially anchor in the layers below $0.8 \mathrm{R}_{\odot}$, and those of the sunspot groups with life times up to 8 days seem to initially anchor in the layers above $0.8 \mathrm{R}_{\odot}$, as suggested by the pattern of the variation in the initial rotation rates of the sunspot groups with their lifetimes 

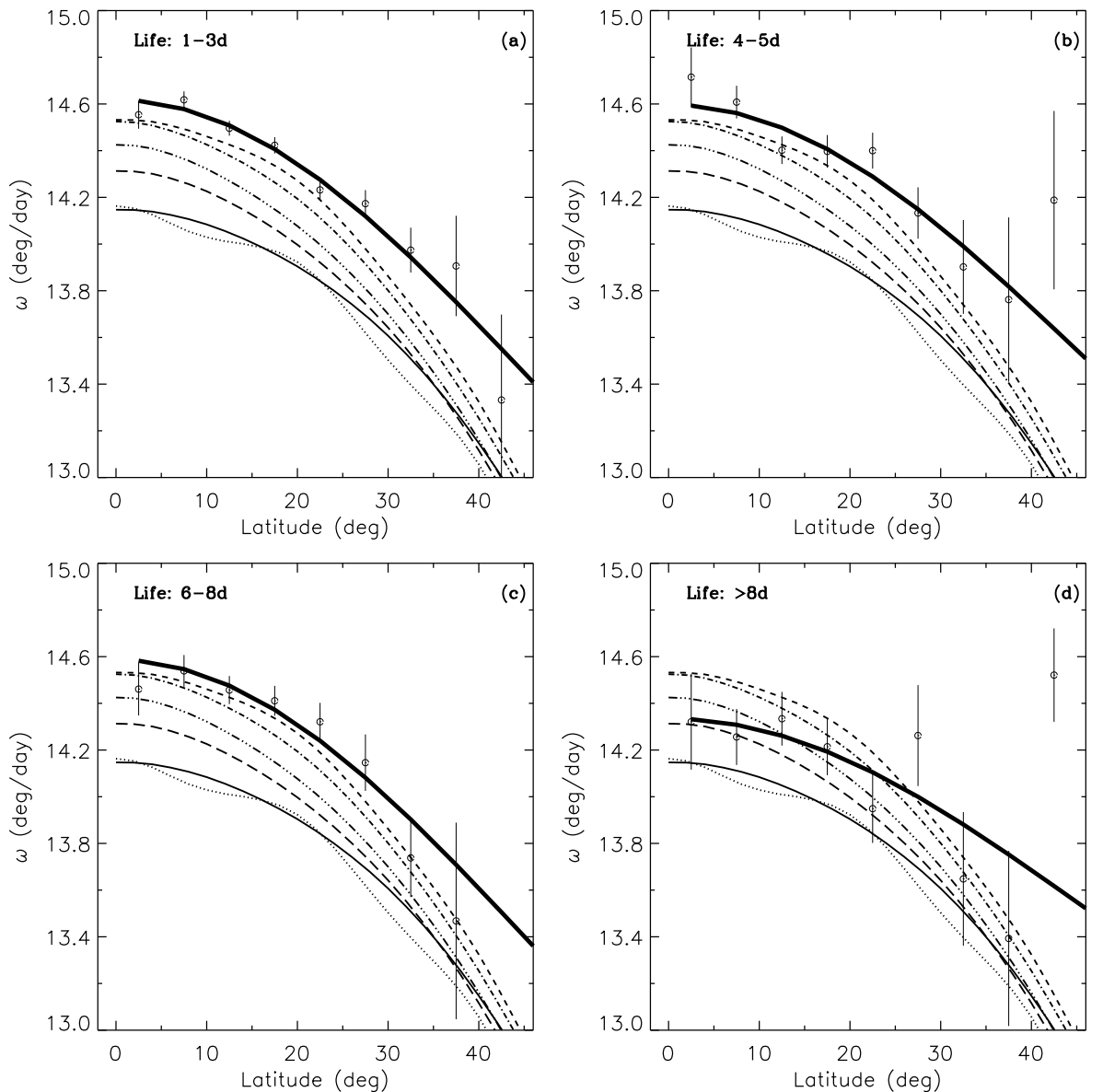

Figure 7. The latitudinal dependence of the "mean initial rotation rates" of sunspot groups whose life times are in the ranges: (a) one-three days, (b) four-five days, (c) six-eight days, and $(\mathrm{d})>$ eight days, determined from the combined Greenwich and SOON data during the period May 1874 -December 2011 by averaging the first day values of $\omega$ over $5^{\circ}$ latitude intervals, $0^{\circ}-5^{\circ}, 5^{\circ}-10^{\circ}, 10^{\circ}-15^{\circ}, \ldots, 45^{\circ}-45^{\circ}$ (plotted at $2.5^{\circ}, 7.5^{\circ}, 12.5^{\circ}, \ldots, 47.5^{\circ}$ ). The error bars represent the standard errors. Here the internal-rotational profiles are correspond to the different depths: thin-solid, long-dashed, three-dotted-dashed, one-dotted-dashed, dashed, and dotted curves represent the profiles at depths $0.75 \mathrm{R}_{\odot}, 0.80 \mathrm{R}_{\odot}, 0.85 \mathrm{R}_{\odot}, 0.90 \mathrm{R}_{\odot}, 0.95 \mathrm{R}_{\odot}$, and $1.0 \mathrm{R}_{\odot}$, respectively. The sunspot-group data corresponding to $\left|D_{\mathrm{C} M}\right| \leq 70^{\circ}$ are used. The thick solid curve represents the corresponding mean profile deduced from the values of the coefficients $A$ and $B$ of Equation (2) obtained from the total number of daily data (i.e. the corresponding values that are given in Figure 6).

(see Javaraiah and Gokhale, 1997b). In Figure 5 the latitudinal dependencies in the mean initial rotation rates of the four different classes of sunspot groups, whose life times in the range one - three, four - five, six - eight, and > eight days, are compared with the latitudinal dependencies in the internal rotation rates at depths $0.75 \mathrm{R}_{\odot}, 0.8 \mathrm{R}_{\odot}, 0.85 \mathrm{R}_{\odot}, 0.9 \mathrm{R}_{\odot}, 0.95 \mathrm{R}_{\odot}$, and $1.0 \mathrm{R}_{\odot}$. As can be seen in this figure, the portions up to $25^{\circ}$ latitude in the profiles of the mean initial rotation 
rates of the shorter than and longer than eight days living sunspot groups are somewhat closer to those of the internal rotation at $0.94 \mathrm{R}_{\odot}-0.96 \mathrm{R}_{\odot}$ and $0.8 \mathrm{R}_{\odot}$, respectively. These results are largely consistent with the results/suggestions in Javaraiah and Gokhale (1997b). (The profile of the very short-lived sunspot groups is even above the rotation profile at $0.94 \mathrm{R}_{\odot}$. The angular motions of super-granules may influence the rotation rates of the very short-lived sunspot groups. In this regard it may be worth noting that the magnetohydrodynamic drag force may be large on the small magnetic structures (D'Silva and Howard, 1994). The results (the profiles of the mean rotation rates of sunspot groups) shown in Figures 2 and 3 are determined from the combined data of all of the sunspot groups during all of the days in their respective lifetimes. These results and also the results (the mean profiles of the initial rotation rates of sunspot groups) shown in Figures $5(\mathrm{a}-\mathrm{c})$ are similar to the results found in most of the other such studies in which the sunspot groups were not sorted out according to their lifetimes (Tuominen, 1962, Tuominen and Virtanen, 1988). Ruždjak et al. (2004) found that the initial velocity of recurrent sunspot groups is larger than the non-recurrent sunspot groups and suggested that the recurrent sunspot groups initially anchor at $0.93 \mathrm{R}_{\odot}$.

The range of the internal rotation rate that corresponds to the increase in depth from $0.94 \mathrm{R}_{\odot}-0.75 \mathrm{R}_{\odot}$ is approximately the same as that corresponding to the decrease in the depth from $0.94 \mathrm{R}_{\odot}-1.0 \mathrm{R}_{\odot}$. In view of the result that the magnetic structures of the sunspot groups with successive longer life times (2-12 days) are initially anchored in successively deeper layers throughout the Sun's convection zone (Javaraiah and Gokhale, 1997b), in Figure 5 the internal rotation rates are shown for a wide range of depths. In Figures 2 and 3 the mean rotation rates of the sunspot groups are compared with the internal rotation rates at a narrow and relatively shallower region, because the average rotation profile of sunspot groups is mostly contributed from the rotation rates of long-lived (lifetime > eight days) sunspot groups during the last few days of their lifetimes (Ruždjak et al., 2004). The rotation rates of the long-lived/large sunspot groups are considerably lower during their initial and final days of their lifetimes.

The derived mean rotation rate of sunspot groups somewhat depends on the cutoff of $D_{\mathrm{C} M}$. It decreases when larger $D_{\mathrm{C} M}$ values are allowed (Ruždjak et al. 2004 and references therein). In all their studies Javaraiah and co-authors allowed $\left|D_{\mathrm{C} M}\right| \leq 75^{\circ}$. Ward (1965, 1966) allowed $\left|D_{\mathrm{C} M}\right|$ up to $80^{\circ}$, whereas many others allowed it up to $60^{\circ}-70^{\circ}$ only. A larger cutoff of $D_{\mathrm{C} M}$ relatively reduces the contributions of long-lived sunspot groups, particularly contributions from during their initial and final days, for the derived mean rotation rate. Hence, the derived value of the mean rotation rate of sunspot groups is relatively large. However, it is necessary to make sure that the first days data sample should not contaminated with the sunspot groups arriving from other side of the Sun. Thus, the calculations of the results shown in Figure 5 are repeated by restricting $D_{\mathrm{C} M}$ up to $\pm 70^{\circ}$ only, and the results are shown in Figures 6 and 7. These results are largely same as those shown in Figure 5. Hence, the conclusions/inferences drawn above from results shown in Figure 5 are largely hold good for the results shown in Figures 6 and 7 also. 
It is widely believed that magnetic flux, in the form of large flux tubes, emerges to the surface presumably from near the base of the convection zone (where the dynamo process is believed to be taking place) and responsible for sunspots and other solar active phenomena (see Rosner and Weiss, 1992, Gough, (2010). There are also suggestions/arguments that the sunspots form just beneath the surface (Kosovichev et al., 2000; Kosovichev, 2002) and in different layers throughout the convection zone (Brandenburg, 2005). The results above are largely consistent with these suggestions/arguments, but the same results also support the idea that large magnetic structures might be generated near the base of the solar convection zone; many of the large magnetic structures may be fragmenting or branching into smaller structures while buoyant rising through the solar convection zone, i.e. small magnetic structures may be fragmented or branched parts of the large magnetic structures (Javaraiah, 2003b). Schüssler and Rempel (2005) argued that the dynamical disconnection of sunspots from their magnetic roots should take place during the final phases of their magnetic structures' buoyant ascent towards the surface.

\subsection{VARIATION IN THE EQUATORIAL ROTATION RATE}

Figure 8 shows the variations in the annual mean values of the equatorial-rotation rate determined from the Doppler-velocity data and the sunspot-group data that did not include the abnormal values of $\omega$. In this figure we also show the variations of the equatorial-rotation rates at $0.96 \mathrm{R}_{\odot}$ and $1.0 \mathrm{R}_{\odot}$ determined from GONG data for each of 147 intervals of three GONG-months during 1995-2009, the variation in the equatorial-rotation rate of the soft X-ray corona determined from the Yohkoh/SXT solar full-disk images for the period 1992-2001 and from the rotation rates of SBCS traced in SOHO/EIT images during the period 1998-2006 (Jurdana-Šepić et al. 2011), and the annual mean sunspot number (Figure 8(b)) to study solar-cycle behavior of the equatorial-rotation rate. Since the mean value $\left(\approx 14.5^{\circ}\right.$ day $\left.^{-1}\right)$ of the equatorial-rotation rate determined from the sunspot-group data during the period $1985-2010$ is close to the mean $\Omega_{0}$ at $0.96 \mathrm{R}_{\odot}(c f$. Figures 2 and 3$)$, the variation in the former is compared with that of the latter, besides comparing it with the variation at $1.0 \mathrm{R}_{\odot}$ (due to the scaling problem the variation in $\Omega_{0}$ at $1.0 \mathrm{R}_{\odot}$ is shown in Figure $8(\mathrm{a})$ instead of in Figure 8(b)). However, as can be seen in Figure 1, the patterns of the variations of the equatorial rotation rates at different depths are largely similar.

As can be seen in Figure 8, the mean value of the equatorial-rotation rate determined from the sunspot-group data is substantially higher (although it is lower during Cycle 22 than in the last 11 Solar Cycles (Javaraiah, Bertello, and Ulrich 2005b; (Suzuki, 2012)) and the amplitude of its variation is also about ten times higher than that determined from the Doppler-velocity measurements. The variation in the equatorial-rotation rate determined from the sunspot-group data steeply decreased during the rising phase of the Solar Cycle 22. It attained minimum at the maximum of this cycle and remained approximately the same level up to the end of the cycle, and then it steeply increased in the beginning of Cycle 23. The overall pattern suggests that there exists a quasi-11-year cycle in the equatorial-rotation rate (correlation coefficient $\approx-0.4$, between 
sunspot number and $A$ ). At the beginning of Cycle 24 the equatorial-rotation rate is considerably higher than that during the last about twenty years (including the beginnings of Cycles 22 and 23). The pattern of the variation in the equatorial-rotation rate is consistent with the well known result of a higher rotation rate during the cycle minimum than during the maximum, found in many studies (e.g. Brajša, Ruždjak, and Wöhl, 2006). However, it should be noted that the minimum years of the solar cycles contain mainly small sunspot groups (Javaraiah, 2012). Small sunspot groups rotate faster than large sunspot groups (e.g. Howard, Gilman, and Gilman, 1984, Javaraiah and Gokhale, 1997b, also see Section 3.1 above). This property of the sunspot groups most probably has an influence on the aforementioned pattern of the Solar Cycle variation in the surface equatorial-rotation rate determined from the sunspot-group data.

The increases of rotational velocities in 2001-2002 and 2008-2009 are most pronounced in GONG results at $1.0 \mathrm{R}_{\odot}$. Similar increases also exist in the result obtained by using sunspot-group data (see Figure 8(a)). In fact, there is a reasonable agreement between the pattern of the variations in the equatorialrotation rates determined from the sunspot-group data and the GONG data $\left(\Omega_{0}\right)$. However, there is a considerable difference in the positions of the minima of these variations, giving an impression of the internal equatorial-rotation rate leads the equatorial-rotation rate of the sunspot groups by one to two years (the reason for this is not known to us).

The annual variation in the equatorial-rotation rate determined from the Doppler-velocity data also steeply decreased during the rising phase and attained minimum at the sunspot maximum of the Solar Cycle 22, but it increased sharply from 1990 to 1993 and suddenly dropped from 1993 to 1994. Overall the equatorial-rotation ration rate is low during Cycle 23, but there is an indication of the variation in the equatorial-rotation rate is in phase with number of sunspots in the interval 1998-2004 (however, the value of $A$ suddenly increased around 2005 and than decreased). This behavior is opposite to that in the previous cycle. The overall pattern of the annual variation indicates the existence of a five to six-year periodicity in the equatorial-rotation rate during Cycle 22.

In Figure 9 the variations in the equatorial-rotation rate determined from the Doppler-velocity data and the sunspot-group data that included the abnormal values of $\omega$ (i.e., $|\omega|>3^{\circ}$ day $^{-1}$ ) calculated by using Equation (3) are compared. As already mentioned above, the inclusion of the abnormal values of $\omega$ increases the uncertainties of the derived coefficients $A$ and $B$, in spite of the size of the data increasing by $4 \%-5 \%$ (the highest is $8.4 \%$ around 2009 ). Hence, with the inclusion of abnormal $\omega$ values the errors $[\sigma]$ of individual yearly averages are so large that they are not significantly (statistically) different from each other so that the variations cannot be examined using them. Therefore, the values of $A$ determined by binning the data into three-year moving time intervals (MTIs) are used. In this figure the variation in $A$ determined from the data that does not include the abnormal values and binned into three-year MTIs is also shown, in order to get an idea about how much difference exists between the yearly (c.f. Figure 8(a)) and three-year MTIs data. In the case of the equatorial-rotation rate determined from the Doppler-velocity data, the three-year smoothed time series obtained from the annual values (c.f. Figure 8(b)) is shown. As can be seen in 

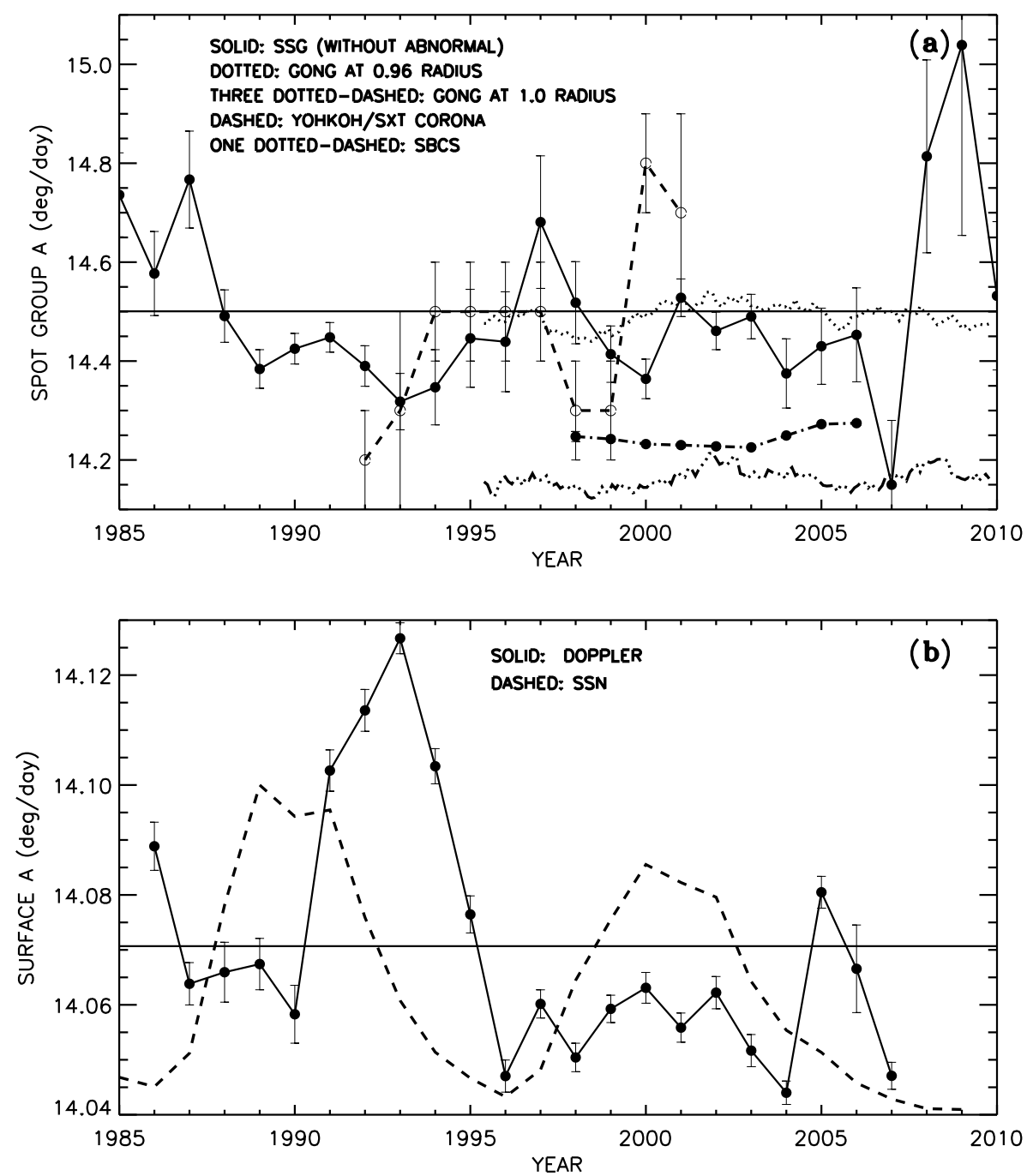

Figure 8. (a) Filled circle-solid curve: the variation in the annual equatorial-rotation rate $A$ determined from the sunspot group (SSG) data after excluding the abnormal values of $\omega$ which are calculated by using Equation (1). Open circle-dashed curve: the variations in the equatorial-rotation rate of the soft X-ray corona determined from Yohkoh/SXT full-disk images for the years $1992-2001$ by Chandra, Vats, and Iyer (2010). Filled circle one dotted-dashed curve: the variation in annual mean equatorial-rotation rate determined from the data of SBCS which were traced in in SOHO/EIT images during the period 1998-2006 (Jurdana-Šepić et al., 2011). In each of these cases the error-bars represent the values of $1 \sigma$ obtained from the linear least-square fits of the data (in the case of SBCS $\sigma$ has very low values). The dotted and three dotted-dashed curves are the variations in the equatorial ration rates at $0.96 R_{\odot}$ and $1.0 R_{\odot}$, determined from GONG data for each of 147 intervals of three GONG-months during 1995-2009 (Antia and Basu, 2010). The solid horizontal line is drawn at the mean values of $14.5^{\circ} \pm 0.2^{\circ}$ day $^{-1}$, determined from the yearly values of the sunspot-group data. (b) The solid curve represents the variation in the annual mean $A$ determined from the corrected Mount Wilson Doppler-velocity data (Javaraiah et al. 2009). The error bars represent one standard error ( $\sigma$ has very high values). The dashed curve represents the variation in the annual mean international sunspot number (SSN), which is normalized to the scale of $A$. The solid horizontal line is drawn at the mean value of $A, 14.07^{\circ} \pm 0.02^{\circ}$ day $^{-1}$, determined from the yearly values. Note: in the case of Mount Wilson Doppler-velocity data in 2007 the data are available only up to March. 
Figure 9, the patterns of the variations in the equatorial-rotation rates derived from the sunspot-group data and the Doppler-velocity data closely resemble one another (correlation coefficient $\approx 0.5$ ). In fact, during Cycle 23 the shapes of the curves are almost the same (the Doppler-velocity measurements are relatively more accurate after 1995). This result indicates that the temporal variation in the equatorial-rotation rate determined from the Doppler-velocity measurements is of solar origin, which has been doubtful so far for the reason mentioned in Section 1. However, this result/conclusion is only suggestive rather than compelling, because the uncertainties in $A$ determined from the sunspot-group data that included the abnormal angular motions of the sunspot groups are large (in the figure the error bar represent one standard error level; the corresponding $\sigma$ is very high). In addition, the abnormal angular motions of the sunspot groups may not represent the angular motions of the surface plasma. These motions may be the abnormal proper motions of the sunspot groups, which took place at the locations of the eruptive solar phenomena such as flares. The Dopplervelocity measurements may be also contaminated with the contributions from such motions of plasma (Note: the spikes in the daily Mt. Wilson velocity data are already removed. Further trimming will create more data gaps and make the data more unrealistic).

On the other hand, the mean values $14.09^{\circ} \pm 0.13^{\circ}$ and $14.21^{\circ} \pm 0.07^{\circ}$ day $^{-1}$ (see Figures 2 and 3 ) of the equatorial-rotation rates determined from the SOON and Greenwich daily sunspot-group data, respectively, which included the abnormal $\omega$ values are significantly (more than $2 \sigma$ level) lower than the corresponding values $14.45^{\circ} \pm 0.008^{\circ}$ and $14.51^{\circ} \pm 0.004^{\circ}$ determined from the data that did not include the abnormal values. Overall it seems that mean values determined from the SOON data that included the abnormal $\omega$ values is somewhat closer to the mean value $\left(14.07^{\circ} \pm 0.02^{\circ}\right.$ day $\left.^{-1}\right)$ of the equatorial-rotation rate determined from the Doppler-velocity data. In Figure 10 (the histogram of the distribution of the abnormal angular velocity values) there is a suggestion that the peaks of the open circle-dashed curve in Figure 9 included the majority of the abnormal velocity values (in the range $10^{\circ}-20^{\circ}$ ) that are somewhat consistent with the rotation rate of the Sun. Therefore, the similarity in the variations of the Doppler-velocity data and the sunspot-group data that included the abnormal motions suggest that the former may be largely of solar origin rather than spurious. Nevertheless, the highly unrealistic latitudinal gradient of the rotation (see Figures 2 and 3 ) that is obtained from the sunspot-group data that included the abnormal $\omega$ values may have a large influence on the corresponding equatorial-rotation rate, making the latter unreliable. For this reason, and because of the large range and the large uncertainties in annual mean values (including all errors of measurements both random and systematic), the reality/reliability of variation in the sunspot-group data that included the abnormal motions is doubtful. Hence, the similarity in the solar-cycle variations in the Doppler-velocity data and the sunspot-group data that included the abnormal motions may rather suggest that variation in the Doppler-velocity data (before 1995) could be contributed by the large uncertainties in this data.

As can be seen in Figure 8, the equatorial-rotation rate determined from the Yohkoh/SXT full-disk images for the period 1992-2001 by Chandra, Vats, and Iyer| 

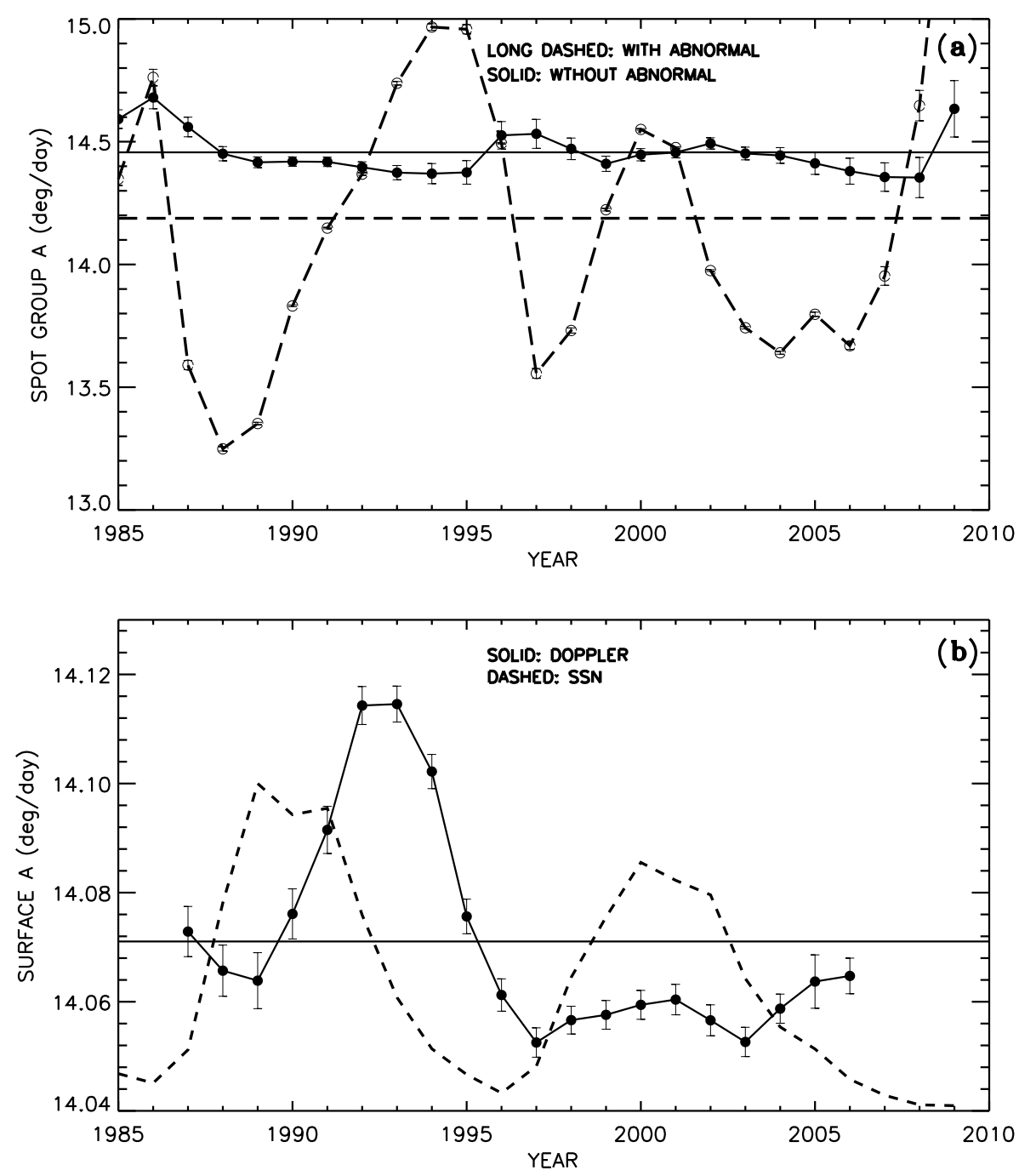

Figure 9. (a) The open circle long-dashed and the filled circle-solid curves represent the variations in $A$ derived from the sunspot group (SSG) data-that included with and without abnormal $\omega$ values $\left(>3^{\circ}\right.$ day $\left.^{-1}\right)$, respectively-in three-year MTIs successively shifted by one year. In the former case the error-bars represent the standard error (because of high $\sigma$ value) and in the letter case they represent the values of $1 \sigma$. The solid and long-dashed horizontal lines are drawn at the corresponding mean values of $A, 14.46^{\circ} \pm 0.09^{\circ}$ day $^{-1}$ and $14.19^{\circ} \pm 0.61^{\circ}$ day $^{-1}$, respectively. (b) The filled circle-solid curve represents the 3 -year smoothed $A$ derived from the annual mean values of $A$ (shown in Figure 8(b)) determined from the Mount Wilson Doppler-velocity data (error bars represent one standard error). The solid horizontal line is drawn at the corresponding mean value of $A, 14.07^{\circ}$ day $^{-1}$. The dashed curve represents the variation in the annual mean international sunspot number (SSN), which is normalized to the scale of $A$. Note: in the case of Mount Wilson Doppler-velocity data in 2007 the data are available only up to March. 


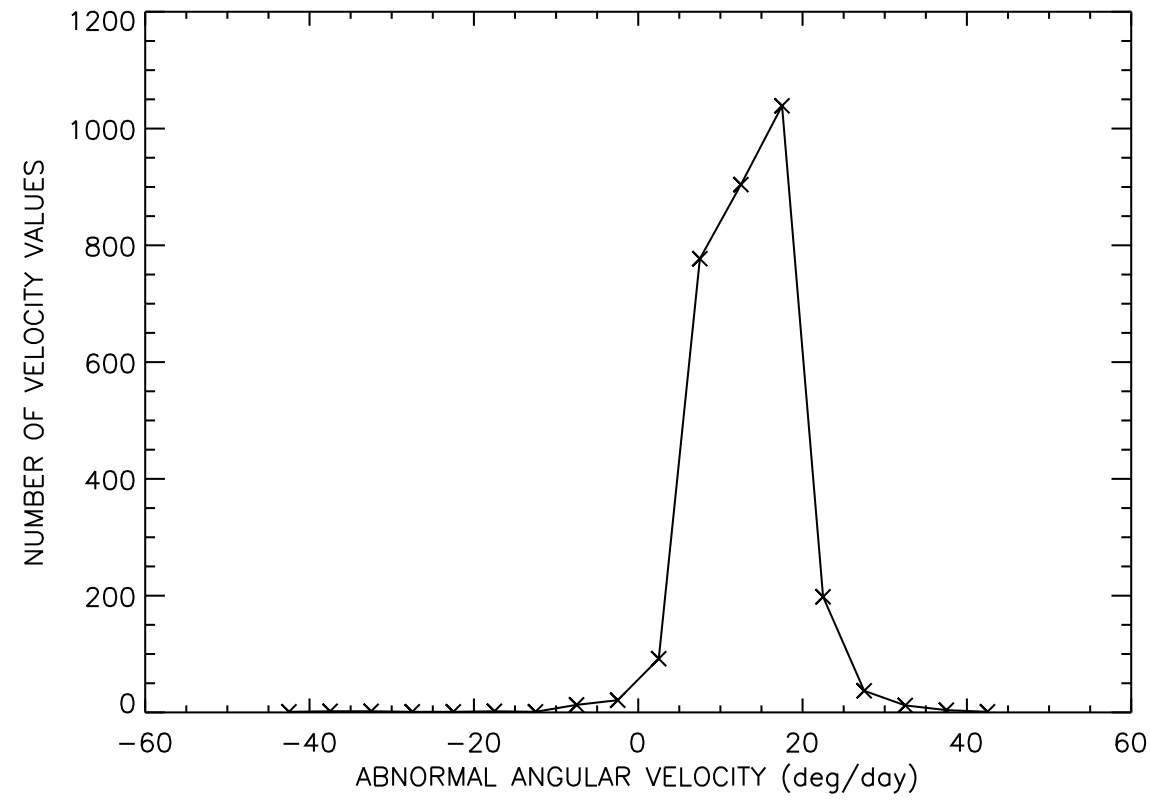

Figure 10. Distribution of the abnormal angular velocity values determined from the SOON sunspot-group data during the period 1 January 1977 to 31 January 2012.

(2010) is closely matches that determined from the sunspot group data for all of the years except for the year 2000, where it has a large value. Close to this year there are humps in the variation of $A$ determined from the sunspot group and the Doppler-velocity data also, although they are small. Thus, the coronal rotation is strongly related to the angular motions of the surface magnetic features. (There seems to be a better agreement between the phases of $\Omega_{0}$ and $A$ of the corona, particularly the agreement is large in minima.) However, the pattern of $A$ determined from the SBCS seems to be considerably different from those of other data sets, particularly in the period 2001-2003. In fact, it seems anticorrelated with that of the GONG data. The reason for this is not known. (It should be noted that the aforementioned similarity between the variation in the equatorial-rotation rate of sunspot groups/corona and the variation in the internal equatorial-rotation rate is only in shape. The amplitude of the variation in the internal equatorial-rotation rate is relatively very small.)

\section{Conclusions}

The following conclusions can be drawn from our analysis:

i) A large portion (up to $\approx 30^{\circ}$ latitude) of the mean differential-rotation profile of the sunspot groups lies between those of the internal differentialrotation rates at $0.94 R_{\odot}$ and $0.98 R_{\odot}$. The portions up to $25^{\circ}$ latitude in 
the mean profiles of the initial rotation rates of the up to eight days and longer than eight days living sunspot groups are close to the those of the internal rotation near $0.96 \mathrm{R}_{\odot}$ and $0.8 \mathrm{R}_{\odot}$, respectively.

ii) At the end of Cycle 23 and the beginning of Cycle 24 the value of the equatorial-rotation rate determined from the sunspot-group data is considerably higher than that of at the beginning of Cycle 23, Overall the pattern of variation in the equatorial-rotation rate during Cycles 22 and 23 resembles the pattern of the known solar-cycle variation in the equatorial-rotation rate determined from sunspot data.

iii) There is a reasonable agreement between the pattern of the variation in the equatorial-rotation rate determined from the sunspot-group data and that determined from the GONG data $\left[\Omega_{0}\right]$. There is also an impression that the variation in the internal equatorial-rotation rate leads the equatorialrotation rate of sunspot groups by one to two years (the reason for this is not known to us).

iv) The pattern of the known solar-cycle variation in the equatorial-rotation rate of the solar corona determined from the Yohkoh/SXT full-disk images and SBCS closely matches with that determined from the sunspot-group data (except during the period 2001-2003). This indicates that the coronal rotation is strongly related to the rotational motion of the surface magnetic features.

v) The variation in the equatorial-rotation rate determined from the Mount Wilson Doppler-velocity data substantially differs from the corresponding variation in the equatorial-rotation rate determined from the sunspot group data that did not include the values of the abnormal angular motions $\left(>\left|3^{\circ}\right|\right.$ day $^{-1}$ ) of the sunspot groups, whereas it closely resembles the corresponding variation determined from the sunspot-group data that included the values of the abnormal angular motions.

vi) Conclusions v) above may suggest that the solar-cycle variation in the surface equatorial-rotation rate determined from the Doppler-velocity measurements (before 1995) is caused by the inconsistency and uncertainties in the data (but it needs further studies to find a definite answer).

Acknowledgments The author thanks the anonymous referee for the detailed comments and useful suggestions, and H.M. Antia for providing the values of the internal-rotation rates that he has determined from GONG data. The author also thanks Luca Bertello for helpful comments and suggestions, B.A. Varghese for his help in making figures, and the organizers of the LWS/SDO-3/SOHO26/GONG2011 workshop for kindly providing a partial financial support to attend the workshop. This work utilizes data obtained by the Global Oscillation Network Group (GONG) Program, managed by the National Solar Observatory (NSO), which is operated by AURA, Inc. under a cooperative agreement with the National Science Foundation. The data were acquired by instruments operated by the Big Bear Solar Observatory, High Altitude Observatory, Learmonth Solar Observatory, Udaipur Solar Observatory, Instituto de Astrofísico de Canarias, and Cerro Tololo Interamerican Observatory. 


\section{References}

Antia, H.M., Basu, S.: 2010, Astrophys. J. 720, 494. doi: 10.1088/0004-637X/720/1/494

Antia, H.M., Basu, S., Chitre, S.M.: 2008, Astrophys. J. 681, 680. doi: $10.1086 / 588523$

Babcock, H.W.: 1961, Astrophys. J. 133, 572. doi: 10.1086/147060

Balthasar, H., Vázquez, M., Wöhl, H.: 1986, Astron. Astrophys. 155, 87.

Brandenburg, A.: 2005, Astrophys. J. 625, 539. doi: $10.1086 / 429584$

Brajša, R., Ruždjak, D., Wöhl, H.: 2006, Solar Phys. 237, 365. doi: 10.1007/s11207-006-0076-7

. Carrington, R.C.: 1863, Observations of the Spots on the Sun, Williams and Norgate.

Chandra, S., Vats, H.O., Iyer, K.N.: 2010, Mon. Not. Roy. Astron. Soc. 407, 1108. doi: 10.1111/j.1365-2966.2010.16947.x

Chou, D.-Y., Fisher, G.H.: 1989, Astrophys. J. 341, 533. doi: 10.1086/167514.

Dikpati, M., Gilman, P.A.: 2006, Astrophys. J., 649, 498. doi: 10.1086/506314

D'Silva, S., Howard, R.F.: 1994, Solar Phys. 151, 213. doi: 10.1007/BF00679072

Foukal, P.: 1972, Astrophys. J. 173, 439. doi: 10.1086/151435.

Godoli, G., Mazzucconi, F.: 1979, Solar Phys. 64, 247. doi: 10.1007/BF00151436

Gough, D.O.: 2010, in Hasan, S.S., Rutten, R.J., (eds.) Magnetic Coupling between the Interior and the Atmosphere of the Sun, Astrophys. Space Sci.Proc. SSA 1570-6591, SpringerVerlag, 37. doi: 10.1007/978-3-642-02859-5_4

Gupta, S.S., Sivaraman, K.R., Howard, R.: 1999, Solar Phys. 188, 225. doi: 10.1023/A:1005229124554

Hiremath, K.M.: 2002, Astron. Astrophys. 386, 674. doi: 10.1051/0004-6361:20020276

Hiremath, K.M., Suryanarayana, G.S.: 2003, Astron. Astrophys. 411, L497. doi: 10.1051/0004-6361:20031618

Howard, R., LaBonte, B.J.: 1980, Astron. Astrophys. 239, L33. doi: 10.1086/183286

Howard, R., Gilman, P.I., Gilman, P.A.: 1984, Astrophys. J. 283, 373. doi: 10.1086/162315

Howe, R., Christensen-Dalsgaard, J., Hill, F., Komm, R.W., Larsen, R.M., Schou, J., Thompson, M.J., Toomre, J.: 2000, Astrophys. J. 533, L163. doi: 10.1086/312623

Javaraiah, J.: 2003a, Solar Phys. 212, 23. doi: 10.1023/A:1022912430585

Javaraiah, J.: 2003b, In: Pevtsov, A.A., Uitenbroek, H. (eds.), Current Theoretical Models and Future High Resolution Solar Observations: Preparing for ATST CS-286, Astron. Soc. Pac., 325.

Javaraiah, J.: 2011, Adv. Space Res. 48, 1032. doi: 10.1016/j.asr.2011.05.004

Javaraiah, J.: 2005, Mon. Not. Roy. Astron. Soc. 362, 1311. doi: 10.1111/j.1365-2966.2005.09403.x

Javaraiah, J.: 2012, Solar Phys., 281, 827. doi: 10.1007/s11207-012-0106-6

Javaraiah, J., Bertello, L., Ulrich, R.K: 2005a, Astrophys. J. 626, 579. doi: 10.1086/429898

Javaraiah, J., Bertello, L., Ulrich, R.K.: 2005b, Solar Phys. 232, 25. doi: 10.1007/s11207-005-8776-y.

Javaraiah, J., Gokhale, M.H.: 1995, Solar Phys. 158, 173. doi: 10.1007/BF00680841

Javaraiah, J., Gokhale, M.H.: 1997a, Solar Phys. 170, 389. doi: 10.1023/A:1004928020737

Javaraiah, J., Gokhale, M.H.: 1997b, Astron. Astrophys. 327, 795.

Javaraiah, J., Gokhale, M.H.: 2002, The Sun's Rotation, Nova Science, New York.

Javaraiah, J., Komm, R.W.: 1999, Solar Phys. 184, 41. doi: 10.1023/A:1005028128077

Javaraiah, J., Ulrich, R.K.: 2006, Solar Phys. 237, 245. doi: 10.1007/s11207-006-0130-5

Javaraiah, J., Ulrich, R.K., Bertello, L., Boyden, J.E.: 2009, Solar Phys. 257, 61. doi: 10.1007/s11207-009-9342-9

Jurdana-Šepić, R., Brajša, R., Wöhl, H., Hanslmeier, A., Poljančić, I., Svalgaard, L., and Gissot, S.F.: 2011, Astron. Astrophys. 534, A17. doi: 10.1051/0004-6361/201014357.

Kambry, M.A., Nishikawa, J.: 1990, Solar Phys. 126, 89. doi: 10.1007/BF00158300

Karak, B.B.: 2010, Astrophys. J. 724, 1021. doi: $10.1088 / 0004-637 X / 724 / 2 / 1021$. Kosovichev, A.G.: 2002, Astron. Nachr., 323, $186 . \quad$ doi: 10.1002/1521-3994(200208)323:3/4〈186::AID-ASNA186〉3.0.CO;2-I

Kosovichev, A.G., Duvall, T.L., Jr., Scherrer, P.H.: 2000, Solar Phys. 192, 159. doi: 10.1023/A:1005251208431

Sivaraman, K.R., Sivaraman, H., Gupta, S.S., Howard, R.: 2003, Solar Phys. 214, 65. doi: 10.1023/A:1024075100667

Meunier, N.: 2005, Astron. Astrophys. 436, 1075. doi: 10.1051/0004-6361:20042414

Meyer, F. Schmidt, H.U., Simon, G.V., Weiss, N.O.: 1979, Astron. Astrophys. 76, 35.

Moreno-Insertis, F.: 1986, Astron. Astrophys. 166, 291. 
Petrovay, K., Brown, J.C., van Driel-Gesztelyi, L., Fletcher, L., Marik, M., Stewart, G.: 1990, Solar Phys. 127, 51. doi: 10.1007/BF00158513

Rosner, R., Weiss, N.O.: 1992, In: Harvey, K.L. (ed.) The Solar Cycle, CS-27,Astron. Soc. Pac., 511.

Ruždjak, D., Ruždjak, V., Brajša, R., Wöhl, H.: 2004, Solar Phys. 221, 225. doi: 10.1023/B:SOLA.0000035066.96031.4f

Schüssler, M., Rempel, M.: 2005, Astron. Astrophys. 441, 337. doi: 10.1051/0004-6361:20052962

Shibata, K., Nozawa, R., Matsumoto, R., Sterling, A.C., Tajima, T.: 1990, Astrophys. J. 351, L25. doi: 10.1086/185671

Suryanarayana, G.S.: 2010, New Astron., 15, 313. doi: 10.1016/j.newast.2009.09.004

Suzuki, M.: 2012, Solar Phys. 278, 257. doi: 10.1007/s11207-012-9946-3

Tuominen, J.: 1962, Z. Astrophys. 55, 110.

Tuominen, I., Virtanen, H.: 1988, Adv. Space Res. 8, 141. doi: 10.1016/0273-1177(88)90183-4

Ulrich, R.K.: 2001, Astrophys. J. 560, 466. doi: 10.1086/322524

Ulrich, R.K., Boyden, J.E.: 2005, Astrophys. J. Lett. 620, L123. doi: 10.1086/428724

van Driel-Gesztelyi, L., Petrovay, K.: 1990, Solar Phys. 126, 285. doi: 10.1007/BF00153051.

Ward, F.: 1965, Astrophys. J. 141, 534. doi: 10.1086/148143

Ward, F.: 1966, Astrophys. J. 145, 416. doi: $10.1086 / 148783$ 